\title{
PREFERENCIAS EN DISTRACCIÓN DE NIÑOS CON CÁNCER
}

\author{
Karol Johanna Briñez Ariza ${ }^{1}$ y Maria Elizabeth Gomez Neva' ${ }^{1}$
}

1Pontificia Universidad Javeriana, Colombia, karolbrineza@javeriana.edu.co; m.gomezn@javeriana.edu.co

\begin{abstract}
Resumo. Introducción: los niños con cáncer reciben intervenciones de enfermería que producen dolor y miedo. Objetivo: describir las preferencias de distracción de niños y adolescentes entre 7 a 16 años con diagnóstico oncológico, en un hospital de Bogotá. Métodos: cualitativo exploratorio descriptivo. Muestreo intencional con 9 participantes de 7 a 16 años con diagnóstico oncológico. Hubo aprobación de comités de ética. Se hicieron entrevistas semiestructuradas, incluyó grabación, observación, notas de campo, transcripción. Etapas: Recolección, Identificación y categorización de descriptores, Análisis de patrones, Temas centrales. Resultados: se obtuvieron 206 descriptores, 11 categorías, y tres temas. El nodo central fue "distracción para mí" que contiene tres explicaciones desde el participante: como le gusta distraerse, lo que ve y piensa en las venopunciones, y desde lo que preferiría en distracción para olvidarse de lo que siente. Conclusiones: las preferencias en tipos de distracción virtual y auditiva de niños son elegir según su gusto: música, series de televisión, películas, juegos virtuales, con audífonos diademas de manos libres. Refirieron que la distracción es importante en las punciones para sentir menos dolor, pensar en otra cosa y salirse de ese ambiente.
\end{abstract}

Palabras clave: Investigación Cualitativa; Enfermería; Preferencias del Paciente; Técnicas.

\section{DISTRACTION PREFERENCES OF CHILDREN WITH CANCER}

Abstract. Introduction: children with cancer diagnosis receive nursing interventions that produce pain and fear. Objective: to describe the distraction preferences of children and adolescents between 7 and 16 years with cancer, in a hospital in Bogotá. Methods: qualitative descriptive exploratory, intentional sampling with 9 participants from 7 to 16 years with cancer diagnosis. There was approval of ethics committees. Semi-structured interviews were conducted, including recording, observation, field notes, transcription. Stages: Collection, Identification and categorization of descriptors, Pattern analysis, Central themes. Results: were obtained 206 descriptors, 11 categories, and three topics. The central node was "distraction for me" that contains three explanations from the participant: how they like to be distracted, what they see and think about the venipunctions, and from what they would prefer in distraction to forget what they feel. Conclusions: the preferences in types of virtual and auditory distraction of children are to choose according to their preferences: music, television series, movies, virtual games, with headphones hands-free headbands. They said that distraction is important in punctures to feel less pain, think about something else and get out of that environment.

Keywords: Qualitative Research; Nursing; Patient Preferences; Techniques.

\section{INTRODUCCIÓN}

El cuidado de enfermería dirigido a niños con diagnóstico oncológico, requiere la aplicación del conocimiento producido por la disciplina con aportes para el mejoramiento de procedimientos habituales de la práctica como es la venopunción frecuente, que es dolorosa y traumática. Se requiere hacer un acercamiento a las preferencias en distracción de niños y adolescentes. La preferencia es la expresión del individuo sobre la conveniencia de una 
acción o la selección entre varias opciones. Es decir, está relacionado con el interés propio, lo que se disfruta, le emociona, gusta, y desea. La distracción implica atraer, disipar y apartar la atención de lo que se vive, en este estudio esa es la cuestión: saber que los distraería mientras los puncionan. Existe un fundamento teórico descrito como la teoría de la distracción, usada en países con conflictos internos políticos donde se distrae la atención del país con otros asuntos internacionales no propios (Niño \& Iregui, 2014). Al vincular teóricamente este fundamento con el presente estudio, se podrá revelar el asunto internacional o externo que un niño o adolescente preferiría para distraerse, mientras ha vivido el conflicto interno de sufrir dolor al ser puncionado. Sin embargo, dada la naturaleza de la teoría de conceptos políticos, y de guerra; y la de este estudio cualitativo en niños, la discusión de los resultados se hará con fundamento empírico derivado de la revisión de literatura científica.

La pregunta de investigación fue: ¿qué preferencias en distracción tienen niños y adolescentes con diagnóstico oncológico? El objetivo fue describir las preferencias de distracción virtual y auditiva de niños y adolescentes entre 7 a 16 años con diagnóstico oncológico en tratamiento con quimioterapia endovenosa, en un hospital de Bogotá, 20182019.

\section{METODOLOGÍA}

Estudio cualitativo con diseño exploratorio descriptivo (Burns \& Grove, 2016) (Figura 1). Muestreo intencional con 9 participantes con edades entre 7 a 16 años con cáncer hospitalizados en el servicio de pediatría del Hospital Universitario San Ignacio de enero a diciembre de 2019. Criterios de exclusión: presencia de infección relacionada con neutropenia febril, infusión de quimioterapia continua o transfusión sanguínea en el momento de la entrevista. Fue aprobado por comité de ética de la Facultad de Enfermería de la Universidad Javeriana y del Hospital. Se usaron consentimiento informado (padres) y asentimiento informado (niños y adolescentes). Recolección de datos: se hicieron entrevistas semiestructuradas con preguntas abiertas en las habitaciones de niños y adolescentes con cáncer asegurando confidencialidad y privacidad, y se inició por una entrevista como inmersión en el campo. La duración osciló entre 6 y 24 minutos. Procedimientos de investigación requeridos: grabación en audio, notas de campo y transcripción fiel a la entrevista por un auxiliar de investigación. El análisis incluyó cuatro etapas: Recolección, descripción y documentación de datos en bruto, Identificación y 
categorización de los descriptores y componentes, Análisis contextual y de patrones, Temas centrales y formulaciones teóricas.

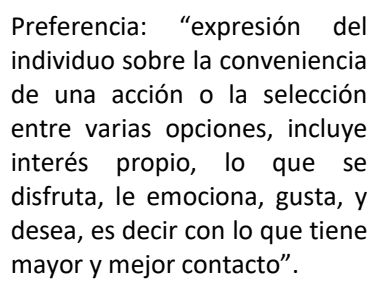

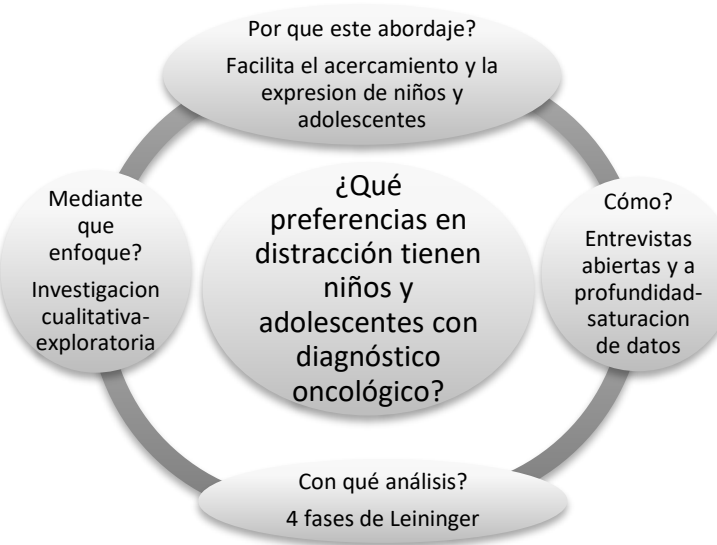

Figura. 1. Coherencia entre lo indagado y la metodología

\section{ANÁLISIS}

De acuerdo a que el proceso de análisis en investigación cualitativa es ecléctico, es decir, no preestablecido, ni único, ni rígido (Morse, 2006; Prado, Souza, Monticelli, Cometto, \& Gómez, 2013; Hernández, Fernández, \& Baptista, 2014) se eligió como guía la propuesta teórica de Madeleine Leininger (Leininger \& McFarland, 2006) para aplicar sus cuatro etapas de análisis, porque facilita describir desde el conocimiento y vivencia de los niños sus preferencias. Para ello se describirán los procesos de inmersión en el campo, el ambiente, la transcripción y por separado cada fase propuesta por esta teórica.

La inmersión en el campo se hizo con la primera entrevista para facilitar la proximidad con el contexto y participantes. Se descubrió que el salón de juegos no podía ser el lugar de las entrevistas porque muchos de los participantes se encontraban en cama con aislamiento y era difícil trasladarlos a espacios en donde se podría poner en riesgo su condición de salud.

\subsection{Transcripción}

La transcripción fue el proceso que siguió a cada entrevista grabada en audio que permitió hacer un análisis exhaustivo del lenguaje al transferir las palabras a un texto en un computador (Hernández, Fernández, \& Baptista, 2014) lo hizo un auxiliar de investigación en Word y se guardo en el programa ATLAS.Ti 8 para el análisis. 


\subsection{Primera fase: Recolección de datos}

La producción de datos siguiente fue la realización de entrevistas hasta encontrar saturación de la información. Tuvieron una duración entre 6 y 24 minutos y se realizaron en horarios de la mañana y la tarde. Previo consentimiento de los padres o cuidadores principales y asentimiento informado del participante firmados, favoreciendo la confidencialidad y privacidad. Una investigadora con experticia en la realización de entrevistas realizaba la formulación de las preguntas mientras la otra investigadora tomaba las notas de campo, y profundizaba algún aspecto que notara que debía ser indagado. Se grabaron en audio. Luego de revisar cada transcripción línea por línea, se codificó cada participante como informante enumerados del 1 a 9 , (informante 1 , informante 2 y así sucesivamente). Se eligieron descriptores de las entrevistas a partir de datos empíricos que se identificaron como significativos en su descripción en cuanto al fenómeno de preferencias en distracción, se hizo revisión por separado por cada investigadora y se hizo reunión para identificar puntos de acuerdo o desacuerdo.

\subsection{Segunda fase: Identificación y categorización de descriptores}

Se inicia el proceso de reducción de datos para extraer y organizar la información de los participantes con el fin de iniciar clasificaciones conceptuales (Creswell, 1998; Hurtado, 2006). En esta fase, se uso el programa atlas ti para almacenamiento, se codificaron los descriptores que corresponden a los registros verbales escritos de los participantes, enumerando por orden cada uno (descriptor 1, descriptor 2). Las entrevistas se codificaron con la letra $E$ y seguido el número que corresponde según la entrevista ( $E 1, E 2 \ldots$ etc.), y adicionalmente se enumeró cada párrafo. Se obtuvieron en total 206 descriptores de las entrevistas a los participantes, los cuales fueron el insumo para el siguiente paso, que consistió en agrupar los descriptores que compartían similitud en su significado, llegando a un segundo nivel de codificación obteniendo codificación axial. Cada categoría obtenida fue leída y mereció un memo que se comprendió como la interpretación del concepto que ese fragmento describía, la etiqueta correspondió a lo expresado por los participantes. De esta segunda fase, se obtuvieron once categorías.

\subsection{Tercera fase: Análisis de patrones}

De las categorías obtenidas de la segunda fase se agruparon las categorías similares mediante comparación constante, identificando patrones recurrentes en tres categorías 
mediante la fase de codificación selectiva. Fue importante en esta etapa, identificar patrones de recurrencia, expresiones y significados que describen las preferencias de distracción de los participantes.

\subsection{Cuarta fase: Temas centrales y formulaciones teóricas}

Se obtuvieron tres temas con un dodo central que se presentarán en los resultados (Tabla 3). Se aplicaron criterios de rigor metodológico: credibilidad, confirmabilidad, significado en contexto, saturación, transferibilidad (Tabla 1) con el fin de demostrar rigurosidad en descubrir la naturaleza del fenómeno estudiado (Leininger \& McFarland, 2006).

Tabla 1. Criterios de rigor metodológico

\begin{tabular}{lc}
\hline \multicolumn{1}{c}{ Criterios } & Aplicación en la investigación \\
\hline Credibilidad & Verdad de los hallazgos \\
Confirmabilidad & Reafirmación con cada participante de lo dicho en cada entrevista \\
$\begin{array}{l}\text { Significado en } \\
\text { contexto } \\
\text { Saturación }\end{array}$ & Inclusión del significado en la distracción de ciertos objetos de los participantes \\
Transferibilidad & El número de entrevistas se determinó hasta que no hubo información nueva ni \\
diferente.
\end{tabular}

\section{RESULTADOS}

\subsection{Ambiente}

El ambiente era la habitación del octavo piso del servicio de pediatría, de paredes blancas, iluminadas con luz natural, cómoda, tranquila, con espacio de descanso en un sofacama para el cuidador y una cama para el participante. Tanto en la inmersión en el campo, como en la producción de datos se tuvo en cuenta que no hubiese otras personas dentro de la habitación para evitar dispersar, interrumpir o afectar la privacidad y confidencialidad de la información que aportaron los participantes.

El uso de las cuatro fases de Leininger, facilitó encontrar un camino sistemático para analizar grandes campos de datos en forma secuencial (Leininger \& McFarland, 2006). Se obtuvieron once categorías de la segunda fase de análisis, que se presentan en la Tabla 2. 
Tabla 2. Segundo nivel de codificación. Categorías obtenidas.

\begin{tabular}{lc}
\hline Categorías obtenidas & Categorías obtenidas \\
\hline No lo distraen a uno & Me distraigo oyendo un cantante preferido \\
Preferencias del dispositivo & Me gustaría ver una serie de televisión \\
Prefiero la música & Técnicas usadas en la práctica \\
Como distraerse & Con auriculares preferiría oír mi música \\
La distracción es importante en las punciones & Distracción para mi es \\
& Combinar lo que oigo y veo \\
\hline
\end{tabular}

\subsection{Temas centrales y formulaciones teóricas}

Esta fase fue la cuarta siguió a la de análisis de patrones, y permitió encontrar como núcleo la categoría "distracción para mi" como eje central, explicada por las tres categorías que contienen tres explicaciones desde como le gusta distraerse al participante, desde lo que ve y piensa este participante en las venopunciones, y desde lo que preferiría en distracción. Es decir, es una interacción entre lo que es el gusto propio, afectado por las cogniciones e imágenes visuales de su experiencia en esos procedimientos, lo anterior, orientaría una idea en el participante de lo que el preferiría cuando lo puncionen, explicado por un concepto de distracción desde el significado de los participantes. Lo anterior se explicita en la Tabla 3.

Tabla 3. Temas centrales y formulaciones teóricas

\begin{tabular}{|c|c|c|c|c|}
\hline & Categorías obtenidas en tercera fase & \multicolumn{2}{|c|}{$\begin{array}{c}\text { Temas obtenidos en } \\
\text { cuarta fase }\end{array}$} & \multirow{2}{*}{$\begin{array}{c}\text { Nodo central } \\
\text { Distracción para }\end{array}$} \\
\hline 1 & Prefiero la música & 1 & "Como me gusta & \\
\hline 2 & Como distraerse & & distraerme" & $\mathrm{mi}$ \\
\hline 3 & Me distraigo oyendo un cantante preferido & & & \\
\hline 4 & Distracción para mi & & & \\
\hline 5 & No lo distraen a uno & 2 & "Cuando me & \\
\hline 6 & Técnicas usadas en la practica & & puncionan" & \\
\hline 7 & La distracción es importante en las punciones & & & \\
\hline 8 & Preferencias del dispositivo & 3 & "Mi preferencia & \\
\hline 9 & Me gustaría ver una serie de televisión & & cuando me & \\
\hline 10 & Con auriculares preferiría oír mi música & & puncionen" & \\
\hline 11 & Combinar lo que oigo y veo & & & \\
\hline
\end{tabular}

\section{DISCUSIÓN}

La preferencia se refiere a la expresión del individuo sobre la conveniencia de una acción o la selección entre varias opciones. Es decir, está relacionado con el interés propio, lo que se disfruta, le emociona, gusta, y desea, es decir con lo que tiene mayor y mejor contacto para distraerse de forma auditiva y virtualmente. Las preferencias de los niños deben ser tenidas en cuenta por los adultos para generar emociones (Flores \& Alcalá, 2018; Organización Panamericana de la Salud, Organización Mundial de la salud \& Centro Latinoamericano y 
del Caribe de Información de Ciencias de la Salud, 2020) estas emociones se genera en el niño al ver que se le tiene en cuenta sus gustos cuando se le va a puncionar, esto lo haría feliz en un ambiente hostil como el hospitalario. Al describir la voz de los niños y adolescentes con cáncer, se identificó que la distracción es un concepto transversal a la investigación, porque sus gustos, lo que viven en la práctica y lo que preferirían giran en torno a lo que para ellos es olvidarse de lo que viven mientras los puncionan.

Al responder a la pregunta planteada en esta investigación debe ligarse el descubrimiento de lo que le gusta a los niños con sus expresiones verbales y el concepto teórico de preferencia de distracción. En este sentido, a partir de los datos obtenidos se encontraron tres temas:

\footnotetext{
"Como me gusta distraerme"- Lo que me gusta, "Cuando me puncionan"- Lo que veo y pienso en las venopunciones, "Mi preferencia cuando me puncionen"-lo que preferiría en distracción cuando me puncionen.

"Como me gusta distraerme"- Lo que me gusta
}

En el primer tema: "Como me gusta distraerme"- Lo que me gusta, se incluyeron las categorías que mencionaron lo que a los niños y adolescentes les gusta para distraerse, estas fueron: Prefiero la música, Como distraerse, Me distraigo oyendo un cantante preferido.

En la categoría "prefiero la música", se identificaron descriptores que revelaron el significado de los gustos, y hablaron las siguientes líneas "Pues a mí casi siempre lo que me distrae a veces es la música, escuchar música pues me distrae de lo demás" (informante 1). La distracción y sus técnicas participan con efectos en las emociones y conducta de los niños (Adler, Schwartz, Waters, \& Stricker, 2016). Es por ello que, en esta investigación, los participantes manifiestan que su sentir se modifica y logran distraerse ante la música. La música podría obstruir una vía secundaria en la corteza prefrontal que participa en la memoria del dolor (Suresh, 2015) además de liberar endorfinas en influencia del sistema nervioso autónomo en la pituitaria (Evans, Tsao, \& Zeltzer, 2008) al tiempo que permite escapar de su propio mundo.

En la categoría "Me distraigo oyendo un cantante preferido", fueron reveladas frases con géneros musicales: "pues me ha gustado siempre, escuchar vallenato" (Informante 1); "es como un tipo de electrónica, pero también cantada" (informante 4); "Pues eso ya sería dependiendo de gustos, pero para mí sería un rock" (informante 5); "me gusta el 
reggaetón, me gusta, mmm, eeh, la música popular" (informante 7), que son escuchados por niños y adolescentes todos los días, escuchando sus éxitos es decir, dicho por ellos "las que son más comunes que escucho" (informante 1).

Lo anterior clarifica que sus preferencias no son de música exclusivamente para jóvenes, sino que incluyen diversos géneros musicales que socialmente son aceptados por sus pares, los oyen en el transporte, en el hogar, en redes sociales, en sus celulares. Siendo coherente, esto plantearía que el efecto podría estar en la presencia de la música, sin que ellos sean exclusivos en sus gustos, lo cual favorecería ante una intervención con música. Incluso, los participantes proponen que sería interesante que en la práctica cuando se les va a hacer una punción venosa existiera un listado para que ellos pudieran elegir y que les ayude a distraerse, se soporta así: "pues tener un listado y uno ir escogiéndolas las que le guste" (informante 1) y "yo la elijo pues yo elegiría una que me entretuviera bien" (informante 5), como contenido definen "diez (10) a quince (15) canciones, pues que no fuera tan largo" (informante 1). De acuerdo a la literatura, oír música es una intervención no farmacológica que puede aliviar el dolor en pediatría y adultos (Adler, Schwartz, Waters, \& Stricker, 2016) esto se ha explicado que mejora su afrontamiento y desvía el significado de la sensación negativa que experimenta.

En la categoría "como distraerse", fueron reveladas frases de elementos propios significativos o cosas que hacen a su edad, constituyendo su preferencia en distracción individual, así lo soportan los siguientes descriptores: "Con los juguetes" (informante 2), "Con una muñeca que, que me regalaron", (informante 2), "viendo algo que me guste" (informante 8). En esta investigación los juguetes propios eran del gusto y han interactuado con ellos, estos aspectos logran hacerlos pensar en otra cosa. El juego es conceptualizado como una acción libre y ejecutada, donde el jugador es absorbido, siente como si fuera de su vida real en un momento y un tiempo (Toro, 2013). Los juguetes en los procesos de hospitalización, son elementos propios y han demostrado efectos positivos. El papel terapéutico del brinquedoterapéutico o juego se describe en cuatro funciones: recreación, estimulación, socialización, catarsis, este se refiere a esa posibilidad que tiene el niño que los usa para liberar tensión emocional y mejorar su afrontamiento. En un estudio (Kiche \& Almeida, 2009), se encontró que hubo comportamientos como postura relajada, juego, expresión facial relajada, y sonrisas, que evidenciaron adaptación y aceptación de los procedimientos en niños expuestos a una intervención con juguetes, en sesiones de brinquedoterapeutico, por lo anterior los juegos pueden distraer. Adicionalmente, hay 
estudios que mencionan la necesidad de su uso en el cuidado de enfermería para favorecer las respuestas de los niños en clinica, aunque no son usados cotidianamente (Bertozzo, Amorim, \& Suzuki, 2012). Su uso en la práctica favorece expresión de sentimientos, disminución de estrés por separación de casa y mayor seguridad en un ambiente extraño (Moura da Cruz, Andrade, Sousa, Luckwü, \& Silva, 2013). El uso de muñecos favorecen la distracción, esto se comprobó por Santiago y colaboradores (Lemos, Oliveira, Gomes, Silva, Silva, \& Fernandes, 2016) al usar el brinquedo terapeutico para punción venosa, porque favoreció que los niños colaboraran espontáneamente al sentirse en un ambiente pediátrico como expresión de su lenguaje natural que es el juego. Otros descriptores de esta categoría hicieron referencia a otras formas de distraerse como viendo televisión o películas, algunos son: "Viendo tele" (informante 3), "Una de Disney", (informante 4). Estas frases revelaron en los ambientes clínicos, hay oportunidad de hacer realidad estos gustos de los niños hospitalizados porque hace parte de lo que comúnmente ellos y sus padres usan para su distracción al usar televisores de las habitaciones, o los celulares o las tablets.

La distracción, justifica que el cerebro es limitado para procesar estímulos dolorosos, por ello al participar en actividades de distracción se limita el enfoque del dolor (Adler, Schwartz, Waters, \& Stricker, 2016). La estrategia elegida para distraer a los niños, responde según la edad, los pequeños responden a juegos o dispositivos y los grandes a estrategias de respiración (Adler, Schwartz, Waters, \& Stricker, 2016). En esta investigación se encontraron frases de participantes sobre los juegos interactivos porque les gustan y los ven en sus dispositivos, además los distrae. Algunos son los siguientes: "cosas de cocina" (informante 4), "Le dan eso, una instrucción a uno de lo que tiene que hacer" (informante 4), "Pues sería como un... un jueguito y que uno tenga que mirar o mover algún mientras tanto la otra manito o el pie" (informante 5). De acuerdo a estos descriptores, los participantes reconocen en el juego interactivo un mundo al que le pueden dan una realidad mediante comandos o manejo manual, esta realidad cercana al mundo que viven, pero de una forma en la que ellos tienen su control desde sus propios dispositivos, lo cual les gusta y les ayuda a distraerse. Por otra parte, la voz materna es otra técnica de distracción que recientemente se comprobó en un estudio en Turquía, donde se encontró que el dolor y frecuencia cardíaca de niños que oían a su madre durante y después de procedimientos dolorosos fueron más bajos que su control (Erdoğan, Turan, \& Pinar, 2020). En esta investigación, se encontró que se reconoce a la madre como elemento significativo para cambiar la 
mentalidad sobre lo que ocurre o concentrarse en otra cosa. Lo anterior apoyado en la siguiente frase: "Simple osea, abrazar a mi mamá y ya. No distraerme más. Porque pues, mmh pues me chuzan y me da miedo, salto y se daña" (informante 8). La mamá fue comprendida para los participantes fuente de refugio, distracción y protección. "Cuando me puncionan"- Lo que veo y pienso en las venopunciones.

El segundo tema de esta investigación fue "Cuando me puncionan"- Lo que veo y pienso en las venopunciones: en esta categoría, se incluyeron las categorías que describieron lo que los niños y adolescentes ven y piensan en las venopunciones en los momentos en los que son puncionados en ambientes clínicos urgencias y hospitalización, estas fueron: "No lo distraen a uno", "Técnicas usadas en la práctica", y "La distracción es importante en las punciones".

Con respecto a las dos primeras, los descriptores que las agruparon explican que en la práctica de enfermería usan técnicas pasivas como respiración o preguntas, sin embargo, refieren que no les ayuda para concentrarse en otra cosa en los momentos de venopunción. Algunas de las frases fueron: "Dicen que tratar de respirar para como calmar el dolor, pero casi no, no sirve" (informante 1), "distraerme en sí, no, me dicen es que respire y que no mire, para como para no tener la impresión de que me están pinchando" (informante 7).

Contrastando estos resultados con la literatura, se encontró que una revisión sistemática mencionó que las intervenciones que han usado técnicas de respiración tienen baja evidencia de su resultado (Birnie, Noel, Chambers, Uman, \& Parker, 2018), acorde con esta investigación los niños y adolescentes manifestaron que cuando la enfermera que los punciona les dice que intenten respirar despacio para calmar el dolor no les ayuda, o que solo les habla y ello no es una técnica de distracción. Como última categoría de este segundo tema fue: "La distracción es importante en las punciones", ésta agrupó frases que hicieron comprender la voz de los niños al hacer un llamado a enfermería para comprender que en las punciones se requieren técnicas que les favorezca pensar en otra cosa, que es un elemento que les permitiría a afrontarlo mejor, porque ellos sienten miedo y angustia y se les debe ayudar a no imbuirse tanto en el dolor o en el momento del procedimiento. Algunos descriptores fueron: "Pues porque, para que se concentren y no tengan mucho dolor" (informante 6), "si no sé, uno se distrae y está es allá en otra cosa y no en lo que le van hacer a uno" (informante 6), "porque también está la gente que le tiene miedo a las agujas" (informante 7). En un estudio sobre representaciones sociales de niños con punciones y 
dolor, se encontró que las punciones hacen sentir en los niños dolor. Este dolor generó sufrimiento que se vio manifestado por llanto y sentimientos de miedo y ansiedad (Guerreiro \& Curado, 2012).

\section{"Mi preferencia cuando me puncionen - lo que preferiría en distracción cuando me puncionen"}

El tercer tema fue "Mi preferencia cuando me puncionen - lo que preferiría en distracción cuando me puncionen": en esta categoría, se incluyeron las categorías que mencionaron lo que niños y adolescentes preferirían para distraerse en las venopunciones, estas fueron: "preferencias del dispositivo", "preferiría ver una serie de televisión", "Con auriculares preferiría oír mi música", "Combinar lo que oigo y veo".

En la primera categoría de este tema "preferencias del dispositivo", se describen las preferencias en distracción visual, que implica el uso de dispositivos que median la interacción activa o pasiva con la técnica de distracción, los informantes mencionaron diferentes colores de acuerdo al género, y el tamaño si se es adolescente unas gafas grandes, esto aseguraría que se sienta que se ha elegido acorde a su preferencia. Algunos descriptores que lo explican fueron: "para las gafas me gustaría un, un azulito como un agua marina o un ... un rosadito, entre rosadito y moradito" (informante 5), "porque voy a pensar que eso está hecho para mi" (informante 5). En un estudio cualitativo sobre distracción positiva con 271 jóvenes de 5 a 19 años en Canadá en espacios de espera de atención pediátrica, se encontró que la edad influyó en las preferencias de la actividad y la ansiedad. Los medios interactivos y los manos libres si incluyeron diversas edades (Biddiss, Knibbe, Fehlings, McKeever, \& McPherson, 2019).

Sumado a la anterior categoría se encontró otra en distracción visual etiquetada como "preferiría ver una serie de televisión", las preferencias fueron diversas opciones de series entre las que están: Dragon ball Z, Ariel de Disney, Pokemon, la pantera rosa, Rapunzel, los increíbles, el hombre araña. Estas preferencias se anidaron a gustos de las edades y genero las femeninas eligen películas tiernas reconocidas y los masculinos las que implican poder y lucha. El desarrollo socioemocional permite a un niño identificar y expresar emociones, cualidades, lo que hace que se auto conozca (Centro de Estudios para el Desarrollo Psicosocial, 2015), tenerle en cuenta estas preferencias haría que se le facilite compartir con otros, reconocerse, tener opiniones, y acogerse a reglas derivadas de sus preferencias, es decir lograr definirse y expresarse para una convivencia en sociedad. Según lo expresado, las preferencias son un término individual que puede ser compartido en características 
generales como que las series de televisión se han estigmatizado por género y sí que al ser niña se ven las de princesas o si se es niño se ven los de superhéroes. Estos tienen un significado especial para esta población, los superhéroes y las princesas tiene una representación de un hombre protector o guerrero y para ellas, implican la estética femenina y la ternura (Alvariñas, \& López, 2018).

Adicionalmente, en este tercer tema la categoría de distracción auditiva: "con auriculares preferiría oír mi música", se encontró que la música mediante auriculares fue el distractor más usado y era cotidiano; por ello a los participantes les gustaría que fuera usado cuando los puncionaran, esto es explicable porque la manipulación de los auriculares la conocen y saben que nos les generará daño. Mencionaron que en sus preferencias para oír la música (componente distractor) preferirían el uso de dispositivos diversos como auriculares, de manos libres y de diademas de tamaño grande con colores llamativos. Algunos descriptores fueron: "Pues a veces cuando tengo el celular y auriculares pues escucho música ahí a ratos" (informante 1); "diademas puede ser" (informante 6); "en el celular pongo los audífonos y siempre uso los manos libres" (informante 8). En un estudio se expuso que el uso de manos libres es además una estrategia que ofrece libertad de movimiento, conexión a varios dispositivos sin cambio de cables (BBC News, 2020). La última categoría de este tema fue "Combinar lo que oigo y veo", y en ella pudo encontrarse que en momentos de punción venosa, los informantes preferirían la posibilidad de distraerse de forma auditiva y visual al mismo tiempo, en sus propias palabras "te vas a desconectar", pero añaden que sabrían que están en una punción, solo que, al emplear las dos técnicas, el resultado es lo que ellos quisieran. Esta técnica combinada se ha descrito que disminuye la incomodidad en procedimientos dolorosos porque controla sensaciones que se estimulan cuando el receptor está viendo o escuchando sobre el procedimiento doloroso (Quiroz \& Melgar, 2012). Los descriptores que apoyan la categoría descrita son: "girando la cabeza para los lados uno ve el, solamente la esta y no, no está pendiente de lo que esta, lo que le están haciendo ahí en, ejemplo cuando es en la mano, sino (informante 1). "puedes estar escuchando música, pero ver la la venita ahí mientras te la cogen eso no te va a ayudar mucho, en cambio si ves, si oyes ya no vas, como que te vas a desconectar de todo lo que te está pasando, pero conscientemente que te van hacer eso, pero te vas a distraer" (informante 5). Nodo central: "La distracción para mí". En la Figura 2 puede identificarse la descripción de las preferencias en distracción virtual y auditiva desde la voz de los participantes. 


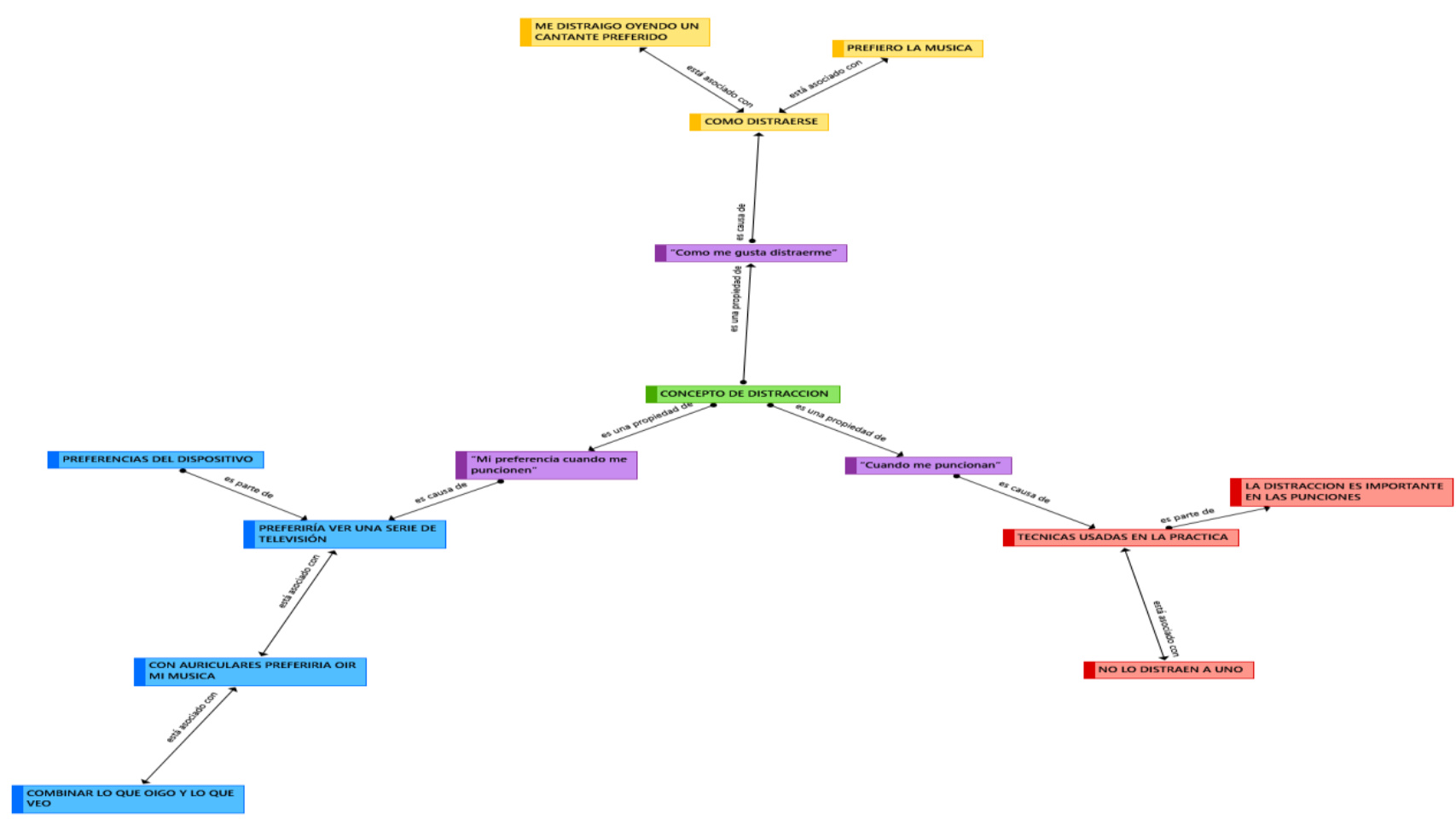

Figura. 2. Las preferencias en distracción virtual y auditiva

El concepto de distracción o "distracción para mí", es nodo central de esta investigación porque está imbuido en cada tema como eje permitiendo que sea comprendido el fenómeno de las preferencias de distracción visual y auditiva. El concepto distracción, está incluido dentro de las intervenciones de enfermería encaminadas al logro de objetivos dentro del proceso de enfermería. Está clasificada con el código 5900 en las Nursing Interventions Classification definida como: el enfoque intencionado de la atención para alejarla de sensaciones indeseables. Es por ello, que dentro de las actividades que pueden realizarse en la práctica de enfermería está, permitirle al niño, o adulto elegir la técnica de distracción que desee, de forma individualizada según lo que le sea efectivo (Casanova et al., 2015). De acuerdo a ello, las técnicas cognitivas (contar o escuchar música) o conductuales (respiración o imágenes) desvían la atención a los dolores de los procedimientos (Adler, Schwartz, Waters, \& Stricker, 2016).

Siendo coherente con este estudio, los participantes expresaron sus preferencias al ser sometidos a una punción venosa periférica, describieron desde el dispositivo hasta lo que preferirían ver y oír. Enfermería tiene el compromiso de intervenir de forma que los sentidos de las personas cuidadas se estimulen para lograr que las sensaciones poco agradables se alejen, o se incentive a que este ser humano que será puncionado piense en otra cosa. En 
esta investigación, se incluyeron participantes con diagnostico oncológico, se analizó que los procedimientos dolorosos implican el desarrollo de una experiencia. La percepción del dolor oncológico no tiene diferencias entre edades ni sexo (Gallego, Martínez, Guerrero, Romero, García, \& Manzano, 2015) pero las preferencias en distracción se derivan de conocimiento, sentimientos y vivencias que rodea la situación de ser venopuncionado.

Para los participantes, el concepto de distracción se describió como olvidarse de la situación actual, soportar, pensar en otra cosa, entretenerse con algo, concentrarse en otra cosa, meterse en algo y creer que va a ser interesante, estar en una cosa, y cambiar los pensamientos o la mentalidad sobre lo que ocurre. En esta investigación, la distracción fue concebida como "olvidarse de lo que está pasando alrededor" (informante 1); aquí el participante describió la importancia de dejar de tener en cuenta la realidad que vive sin que esté presente en su mente. Otro participante refirió: "intentar pensar que es otra cosa, aunque sepa qué es eso" (informante 3), con esta frase el participante mencionó que este concepto es la posibilidad de lograr en su mente ideas o juicios con una intención en algo externo y no en lo que sienta. Otro refirió "como concentrarme en la otra cosa para no sentir dolor" (informante 4), este describió que es la estrategia para bloquear sensaciones y emociones relacionadas con la experiencia negativa de dolor, al reunir en su mente pensamientos sobre algo que no sea la punción.

Según artículos revisados, la distracción es una medida no farmacológica usada para el manejo del dolor en los niños, su meta se logra porque atrae la atención de otros estímulos o situaciones. La clave ha sido demostrar que funciona, hay que aplicarla en la práctica (Boles, 2018).

\section{CONCLUSIONES}

Las preferencias en distracción virtual y auditiva de niños y adolescentes con cáncer son elegir: música, series de televisión, películas, juegos virtuales, con audífonos diademas de manos libres. Refieren que la distracción es importante en las punciones para sentir menos dolor, pensar en otra cosa y salirse de ese ambiente. Se ha demostrado que las técnicas de distracción son benéficas en los procedimientos de dolor, sin embargo, tener en cuenta las preferencias de distracción de los niños y los adolescentes favorece que las intervenciones que realiza enfermería hagan que se mejore su afrontamiento al ser más personalizadas y terapéuticas. 
Implicaciones para la práctica: usar la distracción en las intervenciones de venopunción podría favorecer que los niños y adolescentes piensen en otra cosa y se les desvíe la atención en el dolor. Para la docencia y enfermería: la interacción academia y práctica favorecería que se dé respuesta a fenómenos de cuidado clínicos y se posibiliten otros niveles de investigación, en este caso el experimental en realidad virtual en niños que van a ser puncionados, al tenerlos en cuenta para la intervención.

Para la investigación cualitativa: la inmersión en el campo es un proceso necesario, facilito tomar decisiones sobre el ambiente. La descripción de los fenómenos de cuidado de niños y adolescentes se favorece mediante el acercamiento desde esta al ser abierta exploratoria, y el análisis desde quienes desde su propia voz posibilitaron el aprendizaje sobre las preferencias en distracción, y así motivar intervenciones terapéuticas personalizadas de cuidado de enfermería.

Limitaciones. Seleccionar solo ambiente hospitalario, debería investigarse las preferencias en distracción con una muestra mayor incluyendo ambientes ambulatorios o consulta externa.

\section{REFERENCIAS}

Adler A., Schwartz E., Waters J., Stricker P. (2016). Anesthetizing a child for a large compressive mediastinal mass with distraction techniques and music therapies as the sole agents. Journal of Clinical Anesthesia, 35, 392-397. https://doi.org/10.1016/j.jclinane.2016.09.021

Alvariñas M., López C. (2018). Representación de niñas y niños en imágenes de juguetes: ¿el comienzo del cambio? Cadernos de Pesquisa, 48(167), 310-329. https://doi.org/10.1590/198053144639

BBC News. (2020). [BBC] Ventajas y desventajas de los auriculares inalámbricos [Noticias]. https://elcomercio.pe/tecnologia/gadgets/bbc-ventajas-desventajas-auriculares-inalambricos-161099noticia/

Bertozzo A., de Amorim F., Suzuki D. (2012). Uso rotineiro do brinquedo terapêutico na assistência a crianças hospitalizadas: Percepção de enfermeiros. Acta Paulista de Enfermagem, 25(1), 18-23. https://www.redalyc.org/articulo.oa?id=307023882004

Biddiss E., Knibbe T., Fehlings D., McKeever P., McPherson A. (2019). Positive Distraction in Pediatric Healthcare Waiting Spaces: Sharing Play Not Germs through Inclusive, Hands-Free Interactive Media. Developmental Neurorehabilitation, 22(7), 445-452. https://doi.org/10.1080/17518423.2018.1518351

Birnie K., Noel M., Chambers C., Uman L., Parker J. (2018). Psychological interventions for needle-related procedural pain and distress in children and adolescents. Cochrane Database of Systematic Reviews. https://doi.org/10.1002/14651858.CD005179.pub4

Boles J. (2018). The Powerful Practice of Distraction. Pediatric Nursing, 44(5), 247-253. https://web-b-ebscohostcom.ezproxy.javeriana.edu.co/ehost/pdfviewer/pdfviewer?vid=5\&sid=0bc21283-068d-45f5-b1b1916aa956a5ae\%40sessionmgr101 
Burns N., Grove S. (2016). Investigación en enfermería: Desarrollo de la práctica enfermera basada en la evidencia. Elsevier.

Casanova A., Asensio A., Calvo D., Cobos E., Fernandez Y, et al. (2015). Intervenciones de enfermería nic de utilidad en la atención extrahospitalaria. http://bibliosjd.org/wpcontent/uploads/2017/02/INTERVENCIONES-ENFERMERIA-NIC-UTILES-EN-LA-ATENCIONEXTRAHOSPITALARIA.pdf

Centro de Estudios para el Desarrollo Psicosocial, UNICEF. (2015). Tiempo de crecer: Guía para la familia: el desarrollo de niños y niñas de 4 a 10 años. / autores, CEDEP.

Creswell, J. W. (1998). Qualitative inquiry and research design: Choosing among five traditions. Sage Publications.

Do Prado M., De Souza M., Monticelli M., Cometto M., Gómez P. (2013). Investigación cualitativa en enfermerìa: Metodologìa y Didáctica. Organizaciòn Panamericana de la Salud.

Erdoğan Ç., Turan T., Pinar B. (2020). The effect of maternal voice for procedural pain in paediatric intensive care unit: A randomised controlled trial. Intensive and Critical Care Nursing, 56, 102767. https://doi.org/10.1016/j.iccn.2019.102767

Evans S., Tsao J., Zeltzer L. (2008). Complementary and alternative medicine for acute procedural pain in children. Altern Ther Health Med., 14(5), 52-56. https://www.ncbi.nlm.nih.gov/pubmed/18780584

Flores L., Alcalá G. (2018). Motivación a la lectura y preferencia de los géneros literarios segun el sexo de los estudiantes. Revista Panamericana de Pedagogía: Saberes y Quehaceres del Pedagogo, 26, 141-166. https://eds-a-ebscohost-com.ezproxy.javeriana.edu.co/eds/detail/detail?vid=4\&sid=4786cca4-7347-489a8978-ce423b9ef45a\%40sdc-vsessmgr01\&bdata=Jmxhbmc9ZXMmc2I0ZT1|ZHMtbGI2ZQ\%3d\%3d\#db=fua\&AN=132398625

Gallego C., Martíbnez M., Guerrero N., Romero I., García F., Manzano M. (2015). Manejo del dolor en el paciente oncológico pediátrico y factores que influyen en su percepción. Revista Cubana de Farmacia, 49(2). http://scielo.sld.cu/scielo.php?script=sci_arttext\&pid=S0034-75152015000200017

Guerreiro M., Curado M. (2012). PINCHAR... ¡HACE DAÑO!: Representaciones del dolor en el niño, en edad escolar, sometido a punción venosa. Enfermería Global, 11(25), 58-74. https://doi.org/10.4321/S169561412012000100005

Hernández R., Fernández C. Baptista P. (2014). Metodología de la investigación. McGraw-Hill Education.

Kiche M., Almeida F. (2009). Brinquedo terapêutico: Estratégia de alívio da dor e tensão durante o curativo cirúrgico em crianças. Acta Paulista de Enfermagem, 22(2), 125-130. https://doi.org/10.1590/S010321002009000200002

Leininger M, McFarland M. (2006). Culture care diversity and universality: A worldwide nursing theory. Jones and Bartlett.

LeiningerM., McFarland M. (2006). Culture Care Diversity and Universality: A Worldwide Nursing Theory. Jones \& Bartlett Learning.

Lemos I., Oliveira J., Gomes E., Silva K., Silva P., Fernandes G. (2016). Brinquedo terapêutico no procedimento de punção venosa: Estratégia para reduzir alterações comportamentais. Revista CUIDARTE, 7(1), 1163. https://doi.org/10.15649/cuidarte.v7i1.303

Morse, J. M. (2006). Asuntos críticos en los métodos de investigación cualitativa. Universidad de Antioquia.

Moura da Cruz D., De Andrade N., De Sousa F., Luckwü M., Silva A. (2013). Brinquedo terapêutico: Revisão integrativa. Journal of Nursing UFPE On Line, 7(5), 1443-1448. https://doi.org/10.5205/reuol.3960-314241-SM.0705201325 
Niño, R., Iregui J. (2014). La teoría de la guerra de distracción. Revista Científica General José María Córdova, 12(14), 55-72. http://www.scielo.org.co/pdf/recig/v12n14/v12n14a04.pdf

Organización Panamericana de la Salud, Organización Mundial de la salud, Centro Latinoamericano y del Caribe de Información de Ciencias de la Salud. (2020). Descriptor de la Salud: Preferencia. DeCS. http://decs.bvs.br/cgi-bin/wxis1660.exe/decsserver/

Quiroz J., Melgar R. (2012). Manejo de conducta no convencional en niños: Hipnosis, musicoterapia, distracción audiovisual y aromaterapia: Revisión sistemática. Revista Estomatológica Herediana, 22(2), 129-136. https://www.redalyc.org/articulo.oa?id=421539370009

Suresh S. (2015). Music Eases Kids' Pain after Surgery. Northwest Med Mag, 2(2), 11. https://magazine.nm.org/wp-content/uploads/sites/23/2019/04/15NWMED_Spring_Book-Online-singlepageupdated.pdf

Tójar Hurtado, J. C. (2006). Investigación cualitativa: Comprender y actuar. La Muralla.

Toro F. (2013). Una pieza secreta: Juegos y juguetes en la narrativa de adolfo couve. Revista Chilena de Literatura, 83, 177-195. https://doi.org/10.4067/S0718-22952013000100008 